\title{
DOMINATED PERMUTATIONS OF SUBSEQUENCES OF RANDOM VARIABLES
}

\author{
BY
}

\author{
AARON MEYEROWITZ AND MARK SCHWARTZ
}

\begin{abstract}
The generalized strong law of large numbers of Komlós may be extended to include certain dominated permutations of the random variables. A further extension to larger classes of permutations is obtained through decompositions of sequences of positive integers.
\end{abstract}

1. Introduction. The generalized strong law of large numbers proved by Komlós [3] states that from an $L_{1}$-bounded sequence of random variables $\left(X_{n}\right)$, there exists a subsequence $\left(X_{n}^{\prime}\right)$ and a random variable $X$ in $L_{1}$ such that every further subsequence converges Cesàro a.s. to $X$. When considered in the context of Etemadi's approach $[2]$ to the strong law of large numbers for pairwise independent identically distributed random variables, Komlós' result may be extended to include certain "dominated" permutations of the subsequences. An almost incidental result in this direction is given in [4], where a multiparameter Komlós-type property is considered.

In this paper, we investigate the extent to which the subsequences in the Komlós theorem can be permuted. The main result is the following. If $\left(X_{n}\right)$ is an $L_{1}$ bounded sequence of random variables and $(f(n))$ is an increasing sequence of positive integers, then there exists a subsequence $\left(X_{n}^{\prime}\right)$ and a random variable $X$ in $L_{1}$ such that for any further subsequence $\left(X_{j_{n}}^{\prime}\right)$ and any permutation $\pi$ of the positive integers satisfying $\pi^{-1}(n) \leq f\left(j_{n}\right)$ for each $n \geq 1$, we have

$$
\lim _{n \rightarrow \infty} \frac{1}{n} \sum_{i=1}^{n} X_{j_{\pi(i)}}^{\prime}=X \quad \text { a.s. }
$$

Actually, the result will be shown to hold for an even larger class of permutations. Given a permutation $\sigma$, we can get (1.1) provided that $(\sigma(n))$ decomposes into a finite number of permuted subsequences, each dominated by $f$. A necessary and sufficient condition will be given for such a decomposition.

2. The main result. In the following, we consider a probability space $(\Omega, \mathcal{F}, P)$ and a sequence of random variables $\left(X_{n}\right)$. For $a \geq 0$, define $F_{a}\left(X_{n}\right)=X_{n}$. $I\left(\left|X_{n}\right| \leq a\right)$ to be truncation at the value $a$. Throughout, we will be considering certain admissible permutations of the positive integers. In particular, let $(f(n))$ and $\left(j_{n}\right)$ be increasing sequences of positive integers. A permutation $\pi$ is admissible for $f$ and $\left(j_{n}\right)$ if $\pi^{-1}(n) \leq f\left(j_{n}\right)$ for all $n \geq 1$. Equivalently, the condition may be written $n \leq f\left(j_{\pi(n)}\right)$. We shall call the increasing sequence $f=(f(n))$ a rule.

Received by the editors March 13, 1985.

1980 Mathematics Subject Classification. Primary 60F 15, 60A10.

Key words and phrases. Strong law of large numbers, subsequences, permutations. 
To prove the main theorem, we establish some preliminary results similar to those in [3]. The argument then shifts to apply the ideas in $[\mathbf{2}]$. The chief advantage of the Etemadi approach in obtaining a result about permutations appears to lie in the construction of a subsequence $\left(X_{n}^{\prime}\right)$ whose members are "nearly pairwise orthogonal". On the other hand, Komlós uses martingale difference sequences, which do not accommodate permutations.

LEMMA 2.1. Suppose $\left(X_{n}\right)$ is a sequence of random variables. Let $f$ be a rule and suppose $(G(n))$ is an increasing sequence of positive integers. Then there exists a subsequence $\left(X_{n}^{\prime}\right)$ and a sequence of nonnegative scalars $\left(M_{j}\right)$ such that for any further subsequence $\left(X_{j_{n}}^{\prime}\right)$ and any admissible permutation $\pi$, i.e. $\pi^{-1}(n) \leq f\left(j_{n}\right)$, we have

$$
M_{j} / 2 \leq \int_{j-1<\left|Y_{n}\right| \leq j}\left|Y_{n}\right| d P \leq M_{j}+1 / j^{2}
$$

for $1 \leq n$ and $1 \leq j \leq G(n)$, where $Y_{n}=X_{j_{\pi(n)}}^{\prime}$. Furthermore, if $\sup _{n} E\left|X_{n}\right|<\infty$, then $\sum_{j=1}^{\infty} M_{j}<\infty$.

Proof. For $j \geq 1$, there exists a subsequence $I_{j}$ of $I_{j-1}\left(\right.$ taking $\left.I_{0}=\mathbf{N}\right)$ and $M_{j} \geq 0$ such that for all $n \in I_{j}$

$$
M_{j} / 2 \leq \int_{j-1<\left|X_{n}\right| \leq j}\left|X_{n}\right| d P \leq M_{j}+1 / j^{2} .
$$

For $n \geq 1$, let $i_{n}$ be the $n$th element of $I_{G(f(n))}$. Choose any subsequence $\left(i_{j_{n}}\right)$ and admissible permutation $\pi$. Writing $Y_{n}=X_{i_{j_{\pi(n)}}}$, we have

$$
M_{j} / 2 \leq \int_{j-1<\left|Y_{n}\right| \leq j}\left|Y_{n}\right| d P \leq M_{j}+1 / j^{2}
$$

for $1 \leq n$ and $1 \leq j \leq G\left(f\left(j_{\pi(n)}\right)\right)$. Since $G(n) \leq G\left(f\left(j_{\pi(n)}\right)\right)$, we obtain (2.1). If $\sup _{n} E\left|X_{n}\right|=M<\infty$, then for any $N \geq 1$

$$
\sum_{j=1}^{N} M_{j} \leq 2 \sum_{j=1}^{N} \int_{j-1<\left|Y_{N}\right| \leq j}\left|Y_{N}\right| d P \leq 2 E\left|Y_{N}\right| \leq 2 M .
$$

Thus, $\sum_{j=1}^{\infty} M_{j} \leq 2 M<\infty$.

In the following, we will be considering sequences of random variables which are weakly convergent in $L_{1}$. We use the notation $f_{n} \rightarrow f$ weakly, to mean weak convergence in $L_{1}$; that is, for each $g \in L_{\infty}(\Omega), \lim _{n \rightarrow \infty} \int f_{n} g d P=\int f g d P$.

LEMMA 2.2. Let $\left(X_{n}\right)$ be a sequence of random variables. Then there exists a subsequence $\left(X_{n}^{\prime}\right)$ and a sequence of (bounded) random variables $\left(\beta_{n}\right)$ such that $F_{k}\left(X_{n}^{\prime}\right) \rightarrow \beta_{k}$ weakly in $L_{1}$ for each $k \geq 1$. Furthermore, if $\sup _{n} E\left|X_{n}\right|<\infty$, then there exists $X \in L_{1}$ such that $\beta_{n} \rightarrow X$ a.s. (and in $L_{1}$ ).

PROOF. For each $j \geq 1$, the sequence $\left(F_{j}\left(X_{n}\right)\right)$ is uniformly integrable. So there exists a subsequence $I_{j}$ of $I_{j-1}$ and a random variable $\beta_{j}\left(\left|\beta_{j}\right| \leq j\right.$ a.s. $)$ such that $\lim _{n \in I_{j}} F_{j}\left(X_{n}\right)=\beta_{j}$ weakly in $L_{1}$. Diagonalizing gives the first result. From the 
weak convergence and (2.1), taking $\beta_{0}=0$,

$$
\begin{aligned}
\sum_{k=1}^{\infty} E\left|\beta_{k}-\beta_{k-1}\right| & \leq \sum_{k=1}^{\infty} \frac{\lim }{n} E\left|F_{k}\left(X_{n}^{\prime}\right)-F_{k-1}\left(X_{n}^{\prime}\right)\right| \\
& =\sum_{k=1}^{\infty} \frac{\lim }{n} \int_{k-1<\left|X_{n}^{\prime}\right| \leq k}\left|X_{n}^{\prime}\right| d P \leq \sum_{k=1}^{\infty}\left(M_{k}+1 / k^{2}\right)<\infty
\end{aligned}
$$

if $\sup _{n} E\left|X_{n}\right|<\infty$. Thus, there exists $X \in L_{1}$ such that $\beta_{k} \rightarrow X$ a.s. (and in $L_{1}$ ).

LEMMA 2.3. Suppose $F_{k}\left(X_{n}\right) \rightarrow \beta_{k}$ weakly for each $k \geq 1$, and let $f$ be a rule. Then there exists a subsequence $\left(X_{n}^{\prime}\right)$ such that for every further subsequence $\left(X_{j_{n}}^{\prime}\right)$ and admissible permutation $\pi$,

$$
\begin{gathered}
\left|E\left(F_{j}\left(Y_{n}\right)-\beta_{j}\right) \beta_{j}\right| \leq 1 \text { for } 1 \leq n, 1 \leq j \leq n, \\
\left|E\left(F_{p}\left(Y_{m}\right)-\beta_{p}\right)\left(F_{q}\left(Y_{n}\right)-\beta_{q}\right)\right| \leq 2^{-n} \\
\qquad \text { for } 1 \leq p \leq m, 1 \leq q \leq n, 1 \leq m<n,
\end{gathered}
$$

where $Y_{n}=X_{j_{\pi(n)}^{\prime}}^{\prime}$.

PROOF. By the weak convergence, for each $j \geq 1$ there exists a subsequence $I_{j}$ of $I_{j-1}$ such that $\left|E\left(F_{j}\left(X_{n}\right)-\beta_{j}\right) \beta_{j}\right| \leq 1$ for all $n \in I_{j}$. Let $i_{n}$ be the $n$th element of $I_{f(n)}$. For any subsequence $\left(i_{j_{n}}\right)$ and admissible permutation $\pi$,

$$
\left|E\left(F_{j}\left(Y_{n}\right)-\beta_{j}\right) \beta_{j}\right| \leq 1 \text { for } 1 \leq n, 1 \leq j \leq f\left(j_{\pi(n)}\right)
$$

where $Y_{n}=X_{i_{j_{\pi(n)}}}$. Since $n \leq f\left(j_{\pi(n)}\right)$, we obtain (2.2).

For (2.3), let $X_{1}^{\prime}=X_{1}$ and suppose $X_{i}^{\prime}$ has been determined for $1 \leq i \leq k$. Choose $X_{k+1}^{\prime}$ to satisfy

$$
\left|E\left(F_{p}\left(X_{m}^{\prime}\right)-\beta_{p}\right)\left(F_{q}\left(X_{k+1}^{\prime}\right)-\beta_{q}\right)\right| \leq 2^{-f(k+1)}
$$

for $1 \leq m \leq k, 1 \leq p \leq f(m)$ and $1 \leq q \leq f(k+1)$. The sequence $\left(X_{n}^{\prime}\right)$ is now completely determined. Let $\left(X_{j_{n}}^{\prime}\right)$ be any subsequence and $\pi$ an admissible permutation. Writing $Y_{n}=X_{j_{\pi(n)}}^{\prime}$ and $M(m, n)=\max \left\{f\left(j_{\pi(m)}\right), f\left(j_{\pi(n)}\right)\right\}$, we have

$$
\left|E\left(F_{p}\left(Y_{m}\right)-\beta_{p}\right)\left(F_{q}\left(Y_{n}\right)-\beta_{q}\right)\right| \leq 2^{-M(m, n)}
$$

for $1 \leq p \leq f\left(j_{\pi(m)}\right), 1 \leq q \leq f\left(j_{\pi(n)}\right)$ and $1 \leq m<n$. Since $\pi$ is admissible, we get (2.3).

The next lemma uses the preceding results, and is the last step before the main theorem.

LEMMA 2.4. Suppose $\sup _{n} E\left|X_{n}\right|<\infty, F_{k}\left(X_{n}\right) \rightarrow \beta_{k}$ weakly for each $k \geq 1$, and let $f$ be a rule. Then there exists a subsequence $\left(X_{n}^{\prime}\right)$ such that for any further subsequence $\left(X_{j_{n}}^{\prime}\right)$ and admissible permutation $\pi$,

$$
\begin{gathered}
\sum_{n=1}^{\infty} P\left(\left|X_{j_{\pi(n)}}^{\prime}\right|>n\right)<\infty, \\
\sum_{n=1}^{\infty} E\left(Z_{n}^{2}\right) / n^{2}<\infty,
\end{gathered}
$$




$$
\sum_{m \neq n}\left|E\left(Z_{m} Z_{n}\right)\right|<\infty
$$

where $Z_{n}=F_{n}\left(X_{j_{\pi(n)}}^{\prime}\right)-\beta_{n}$.

ProOF. For a given rule $f$, pass to a subsequence $\left(X_{n}^{\prime}\right)$ satisfying $(2.1),(2.2)$ and (2.3). Take $(G(n))$ to satisfy $\sum 1 / G(n)<\infty$. Let $\left(X_{j_{n}}^{\prime}\right)$ be a subsequence and $\pi$ an admissible permutation. Writing $Y_{n}=X_{j_{\pi(n)}}^{\prime}$, we have from (2.1)

$$
\begin{aligned}
\sum_{n=1}^{\infty} P\left(\left|Y_{n}\right|>n\right) & =\sum_{n=1}^{\infty} P\left(n<\left|Y_{n}\right| \leq G(n)\right)+\sum_{n=1}^{\infty} P\left(\left|Y_{n}\right|>G(n)\right) \\
& \leq \sum_{n=1}^{\infty} \sum_{i=n+1}^{G(n)} \frac{1}{i-1} \int_{i-1<\left|Y_{n}\right| \leq i}\left|Y_{n}\right| d P+\sum_{n=1}^{\infty} \frac{E\left|Y_{n}\right|}{G(n)} \\
& \leq \sum_{n=1}^{\infty} \sum_{i=n+1}^{\infty} \frac{1}{i-1}\left(M_{i}+\frac{1}{i^{2}}\right)+K \\
& \leq \sum_{i=2}^{\infty}\left(M_{i}+\frac{1}{i^{2}}\right)+K<\infty, \quad \text { which is (2.4). }
\end{aligned}
$$

For (2.5),

$$
\begin{aligned}
\sum_{n=1}^{\infty} \frac{E\left(Z_{n}^{2}\right)}{n^{2}} & \leq \sum_{n=1}^{\infty} \frac{E\left(F_{n}\left(Y_{n}\right)^{2}\right)}{n^{2}}+2 \sum_{n=1}^{\infty} \frac{\left|E\left(F_{n}\left(Y_{n}\right)-\beta_{n}\right) \beta_{n}\right|}{n^{2}} \\
& \leq \sum_{n=1}^{\infty} \sum_{i=1}^{n} \frac{1}{n^{2}} \int_{i-1<\left|Y_{n}\right| \leq i} Y_{n}^{2} d P+2 \sum_{n=1}^{\infty} \frac{1}{n^{2}} \quad \text { (using (2.2)) } \\
& \leq \sum_{n=1}^{\infty} \sum_{i=1}^{n} \frac{i}{n^{2}}\left(M_{i}+\frac{1}{i^{2}}\right)+K \\
& \leq c \sum_{i=1}^{\infty}\left(M_{i}+\frac{1}{i^{2}}\right)+K<\infty
\end{aligned}
$$

Finally, applying (2.3)

$$
\begin{aligned}
\sum_{m \neq n}\left|E\left(Z_{m} Z_{n}\right)\right| & =2 \sum_{m=1}^{\infty} \sum_{n>m}\left|E\left(Z_{m} Z_{n}\right)\right| \\
& \leq 2 \sum_{m=1}^{\infty} \sum_{n>m} 2^{-n} \leq 2 \sum_{m=1}^{\infty} 2^{-m}<\infty
\end{aligned}
$$

which is $(2.6)$.

We are now ready for the main theorem. The idea behind the proof is the following. With no loss of generality, we assume $X_{n} \geq 0$. The Borel-Cantelli lemma gives Cesàro convergence along any lacunary subsequence, and monotonicity of the partial sums gives the final result.

THEOREM 2.5. Suppose $\sup _{n} E\left|X_{n}\right|<\infty$ and let $f$ be a rule. Then there exists a subsequence $\left(X_{n}^{\prime}\right)$ and a random variable $X \in L_{1}$ such that for any further 
subsequence $\left(X_{j_{n}}^{\prime}\right)$ and admissible permutation $\pi$,

$$
\lim _{n \rightarrow \infty} \frac{1}{n} \sum_{i=1}^{n} X_{j_{\pi(i)}}^{\prime}=X \quad \text { a.s. }
$$

Proof. Since $\left(X_{n}^{+}\right)$and $\left(X_{n}^{-}\right)$satisfy the hypothesis of the theorem, it is sufficient to consider the case $X_{n} \geq 0$. Let $\left(X_{n}^{\prime}\right)$ and $X$ be those given by Lemmas 2.2 and 2.4. Let $\left(X_{j_{n}}^{\prime}\right)$ be any subsequence and $\pi$ an admissible permutation. Define

$$
S_{n}=\sum_{i=1}^{n} X_{j_{\pi(i)}}^{\prime}, \quad S_{n}^{*}=\sum_{i=1}^{n} F_{i}\left(X_{j_{\pi(i)}}^{\prime}\right) \quad \text { and } \quad T_{n}=\sum_{i=1}^{n} \beta_{i} .
$$

Choose $\varepsilon>0$ and $\alpha>1$. Writing $k_{n}=\left[\alpha^{n}\right]$, we have

$$
\begin{aligned}
\sum_{n=1}^{\infty} P\left(\left|S_{k_{n}}^{*}-T_{k_{n}}\right|>k_{n} \varepsilon\right) & \leq c \sum_{n=1}^{\infty} \frac{1}{k_{n}^{2}} E\left(S_{k_{n}}^{*}-T_{k_{n}}\right)^{2} \\
& \leq c \sum_{n=1}^{\infty} \frac{1}{k_{n}^{2}} \sum_{i=1}^{k_{n}} E Z_{i}^{2}+c \sum_{n=1}^{\infty} \frac{1}{k_{n}^{2}} \sum_{i \neq m}\left|E\left(Z_{i} Z_{m}\right)\right| \\
& \leq c \sum_{i=1}^{\infty} \frac{E Z_{i}^{2}}{i^{2}}+c \sum_{n=1}^{\infty} \frac{1}{k_{n}^{2}}<\infty
\end{aligned}
$$

by (2.5) and (2.6), where $c$ is a constant, possibly different at each appearance, and $Z_{n}=F_{n}\left(X_{j_{\pi(n)}}^{\prime}\right)-\beta_{n}$.

By the Borel-Cantelli lemma, $\left(S_{k_{n}}^{*}-T_{k_{n}}\right) / k_{n} \rightarrow 0$ a.s. By (2.4) and Lemma 2.2 we get $S_{k_{n}} / k_{n} \rightarrow X$ a.s. Now, by monotonicity of $\left(S_{n}\right)$,

$$
\frac{1}{\alpha} X \leq \lim _{n} \frac{S_{n}}{n} \leq \varlimsup_{n} \frac{S_{n}}{n} \leq \alpha X \quad \text { a.s. }
$$

Since this holds for all $\alpha>1$, we conclude $S_{n} / n \rightarrow X$ a.s.

Under certain conditions, the limit $X$ in Theorem 2.5 can be more precisely identified. If $X_{n} \rightarrow Y$ weakly in $L_{1}$, then it can be shown that $\beta_{n} \rightarrow Y$ a.s. in Lemma 2.2. Consequently, the limit in Theorem 2.5 is $Y$ and is the only possible limit.

3. Extension of the result. Theorem 2.5 can be extended to a larger class of permutations. Let $\left(X_{n}^{\prime}\right)$ be the subsequence obtained there and let $\left(X_{j_{n}}^{\prime}\right)$ be any further subsequence. For any permutation $\pi$, define $\left(a_{n}\right)=\left(j_{\pi(n)}\right)$. Recall, $\pi$ is admissible (for $f$ and $\left(j_{n}\right)$ ) if $n \leq f\left(a_{n}\right)$ for all $n$. The conclusion of Theorem 2.5 holds if the condition that $\pi$ be admissible is replaced by the weaker requirement that $\left(a_{n}\right)$ decompose into a finite number of subsequences $\left(a_{i, n}\right), i=1, \ldots, k$, which satisfy $n \leq f\left(a_{i, n}\right)$ for all $n$. After establishing terminology, we give a necessary and sufficient condition for such a decomposition and close with an example.

Let $f$ be a rule and consider arbitrary sequences $\left(a_{n}\right)$ of distinct positive integers. Define $A(f)$ to be the set of infinite and finite admissible sequences for $f$. That is, $A(f)=\left\{\left(a_{n}\right): n \leq f\left(a_{n}\right)\right.$ for all $\left.n \geq 1\right\}$. If the terms of a sequence $\left(a_{n}\right)$ are partitioned into sets $S_{1}, \ldots, S_{k}$ and $\left(a_{i, n}\right)$ is the sequence of terms in $S_{i}$, ordered as in $\left(a_{n}\right)$, then we write $\left(a_{n}\right)=\bigcup_{i=1}^{k}\left(a_{i, n}\right)$ and call the collection $\left\{\left(a_{i, n}\right)\right\}$ a 
decomposition of $\left(a_{n}\right)$. Let $A_{k}(f)=\left\{\left(a_{n}\right):\left(a_{n}\right)=\bigcup_{i=1}^{m}\left(a_{i, n}\right)\right.$, where $\left(a_{i, n}\right) \in A(f)$ for $1 \leq i \leq m \leq k\}$. So $A_{k}(f)$ is the set of sequences having a decomposition into $k$ or fewer subsequences in $A(f)$. We describe an algorithm which decomposes any given sequence into as few subsequences in $A(f)$ as possible (maybe infinitely many). This provides a somewhat impractical test for determining whether a particular sequence finitely decomposes, but leads to a necessary and sufficient condition on any other rule $g$ to have $A(g) \subset \bigcup_{k=1}^{\infty} A_{k}(f)$.

Consider the set of ordered pairs of integers with the ordering $(i, j)<(k, l)$ if $i<k$ or $i=k$ and $j<l$. In the decomposition algorithm, we consider the terms of a sequence $\left(a_{n}\right)$ successively, assigning $a_{i, j}=a_{n}$, where $(i, j)$ is the least unassigned pair (in the ordering above) with $j \leq f\left(a_{n}\right)$. In other words, each term $a_{n}$ is adjoined to the end of the first (partial) subsequence which it does not force out of $A(f)$. Denote this process and the resulting decomposition by $M$ (minimal); we show that $M$ is best possible.

LEMMA 3.1. If $\left(a_{n}\right)$ has a decomposition into $k \geq 1$ subsequences in $A(f)$, then $M$ decomposes $\left(a_{n}\right)$ into $k$ or fewer subsequences in $A(f)$.

PROOF. Suppose there exists a decomposition $Q$ of $\left(a_{n}\right)$ into $k \geq 1$ subsequences in $A(f)$. We show by induction on $m \geq 1$ that there is a modification of $Q$ into $k$ subsequences in $A(f)$ which agrees with decomposition $M$ for $a_{1}, \ldots, a_{m}$. Thus, $M$ assigns every term of $\left(a_{n}\right)$ to one of $k$ or fewer subsequences in $A(f)$.

Denote the terms of $Q$ by $a_{i, j}=a_{n(i, j)}, i, j \geq 1$, and suppose $Q$ agrees with $M$ in the terms $a_{1}, \ldots, a_{m-1}$. Assume $Q$ assigns $a_{i, j}=a_{m}$ and $M$ assigns $a_{p, q}=a_{m}$. By definition of $M, p \leq i$ and $q \geq j$. If $p=i$, then $q=j$ and there is nothing to show, so assume $p<i$. We distinguish three cases, and in each, alter $Q$ to obtain a new decomposition into subsequences $\left(a_{i, j}^{\prime}\right)$ which agrees with $M$ for the terms $a_{1}, \ldots, a_{m}$.

(i) If $a_{p, q}$ is never assigned a value by $Q$, define $a_{p, q}^{\prime}=a_{m}$ and $a_{i, r}^{\prime}=a_{i, r+1}$ for $r \geq j$.

(ii) If $Q$ assigns $a_{p, q}=a_{n}$ (so $m<n$ ), then let $u=\max \{t \geq j: n(i, t)<n\}$. If $u \leq q$, then define $a_{p, q}^{\prime}=a_{m}, a_{i, r}^{\prime}=a_{i, r+1}$ for $j \leq r \leq u-1$, and $a_{i, u}^{\prime}=a_{n}$.

(iii) If $u>q$, then define $a_{p, q}^{\prime}=a_{m}, a_{i, r}^{\prime}=a_{i, r+1}$ for $j \leq r \leq q-1, a_{i, q}^{\prime}=a_{n}$, and $a_{p, r}^{\prime}=a_{i, r}$ and $a_{i, r}^{\prime}=a_{p, r}$ for $r>q$.

In each of the cases, $a_{s, t}^{\prime}=a_{s, t}$ for any pair $(s, t)$ not mentioned. It is easy to check that the resulting decomposition satisfies the requirements, thus completing the proof.

EXAMPLE 3.2. Let $f(n)=n^{2}$ and consider $\left(a_{n}\right)=(7,10,1,3,5,2,4)$. By $M$ we get

$$
\begin{aligned}
& 7,10,3,5,4 \\
& 1,2
\end{aligned}
$$

For the main result we define certain "iterates" of $f$. Let $f_{1}(n)=f(n)+1$ and $f_{k}(n)=f\left(f_{k-1}(n)\right)+f_{k-1}(n)$ for $k>1$. Similarly, take $\hat{f}_{0}(n)=n$ and $\hat{f}_{k}(n)=f\left(\hat{f}_{k-1}(n)\right)+\hat{f}_{k-1}(n)$ for $k>0$. Note that $\hat{f}_{k}(n)>f_{k}(n)>\hat{f}_{k-1}(n)$ for all $k>0, n>1$. For example, if $f(n)=n$, then $\hat{f}_{k}(n)=2^{k} n$ while $f(n)=n^{2}$ gives $\hat{f}_{k}(n)=n^{2^{k}}+$ lower order terms. 
THEOREM 3.3. Let $f$ and $g$ be rules. Then $A(g) \subset \bigcup_{j=1}^{\infty} A_{j}(f)$ if and only if there exists $k \geq 1$ such that $g(n)<\hat{f}_{k}(n)$ for all $n \geq 1$.

PROOF. Given that $g(n)<\hat{f}_{k}(n)$ for all $n \geq 1$, we show that $A(g) \subset A_{k+1}(f) \subset$ $\bigcup_{j=1}^{\infty} A_{j}(f)$. Suppose $\left(a_{n}\right) \notin A_{k+1}(f)$. Under algorithm $M$, there exists $i \geq 1$ such that $a_{k+2,1}=a_{i}$. For $1 \leq m \leq k+1$ define $J_{m}=\max \left\{j: a_{m, j}\right.$ is assigned by $M$ from among $\left.a_{1}, \ldots, a_{i-1}\right\}$. By induction on $0 \leq p \leq k$ and the definition of $M$, it must be the case that $\max \left\{a_{k+1-p, j}: 1 \leq j \leq J_{k+1-p}\right\} \geq f_{p+1}\left(a_{i}\right)$. Consequently, $i \geq f_{k+1}\left(a_{i}\right) \geq \hat{f}_{k}\left(a_{i}\right)>g\left(a_{i}\right)$, and so $\left(a_{n}\right) \notin A(g)$.

For the reverse implication, assume that for each $k \geq 1$ there exists $n_{k} \geq 1$ such that $g\left(n_{k}\right) \geq \hat{f}_{k}\left(n_{k}\right)$. Without loss of generality, we can assume $n_{k}>\hat{f}_{k-1}\left(n_{k-1}\right)$ for each $k>1$. We show that the sequence

$$
\begin{aligned}
\left(a_{m}\right) & =\left(\hat{f}_{1}\left(n_{1}\right), \hat{f}_{1}\left(n_{1}\right)-1, \ldots, n_{1}+1, n_{1}, \hat{f}_{2}\left(n_{2}\right), \ldots, n_{2}, \ldots\right) \\
& \in A(g) \backslash \bigcup_{j=1}^{\infty} A_{j}(f) .
\end{aligned}
$$

For any $n$ satisfying $n_{k} \leq n \leq \hat{f}_{k}\left(n_{k}\right)$, let $j=\operatorname{index}$ of $n$ and $i=\operatorname{index}$ of $n_{k}$ in the sequence $\left(a_{m}\right)$. Then

$$
j \leq i=\sum_{p=1}^{k}\left(\hat{f}_{p}\left(n_{p}\right)-n_{p}+1\right) \leq \hat{f}_{k}\left(n_{k}\right) \leq g\left(n_{k}\right) \leq g(n),
$$

and so $\left(a_{m}\right) \in A(g)$. Now for each $k \geq 1$, the algorithm $M$ decomposes the sequence $\left(\hat{f}_{k}\left(n_{k}\right), \ldots, n_{k}\right)$ into $\bigcup_{i=1}^{k+1}\left(a_{i, m}\right)$, where $a_{k+1,1}=n_{k}$. Thus $\left(a_{m}\right) \notin \bigcup_{j=1}^{\infty} A_{j}(f)$, completing the proof.

Note that if $A(g) \subset \bigcup_{j=1}^{\infty} A_{j}(f)$, i.e. if every admissible sequence for $g$ can be finitely decomposed into admissible subsequences for $f$, then in fact $A(g) \subset A_{k}(f)$ for some $k \geq 1$. Consequently, $A(g)$ is uniformly finitely decomposable.

We give an example relating this section to Theorem 2.5 .

EXAMPLE 3.4. Let $\sup _{n} E\left|X_{n}\right|<\infty$ and $f(n)=n$. Then there exists a subsequence $\left(X_{n}^{\prime}\right), X \in L_{1}$, such that for any further subsequence $\left(X_{j_{n}}^{\prime}\right)$, any constant $c \geq 1$ and any permutation $\pi$ satisfying $\pi^{-1}(n) \leq c n$, we have

$$
\lim _{n \rightarrow \infty} \frac{1}{n} \sum_{i=1}^{n} X_{j_{\pi(i)}}^{\prime}=X \quad \text { a.s. }
$$

This follows from the sact that $\hat{f}_{k}(n)=2^{k} n>c n$ for $k$ sufficiently large. Similarly, if $f(n)=n^{2}$ or $f(n)=2^{n}$, then (3.1) is guaranteed to hold for any polynomially or exponentially bounded permutation, respectively. Here, $n^{2}$ and $2^{n}$ may be replaced by $n^{\alpha}$ and $\alpha^{n}$ for $\alpha>1$, with appropriate rounding to integers.

There are certain cases not covered by Theorem 3.3. For $f(n)=n$, the sequence

$$
\sigma(n)= \begin{cases}n, & n \neq 2^{k}, 3^{k} \text { for } k>1 \\ 3^{k}, & n=2^{k} \\ 2^{k}, & n=3^{k}\end{cases}
$$

decomposes into two subsequences, the second being $\left(2^{n}\right)$, and so (3.1) will hold for $j_{n}=n$ and $\sigma$. However, given any rule $g$ for which $(\sigma(n))$ is admissible, we have $A(g) \not \subset \bigcup_{j=1}^{\infty} A_{j}(f)$. 
Finally, the question arises whether a Komlós-type theorem can be obtained for arbitrary permutations. It does not appear that the method of proof given here can provide such a result. Aldous [1] approaches the subject of limit theorems for subsequences through exchangeable sequences and uniformity arguments which could provide insight to the problem.

\section{REFERENCES}

1. D. J. Aldous, Limit theorems for subsequences of arbitrarily-dependent sequences of random variables, Z. Wahrsch. Verw. Gebiete 40 (1977), 59-82.

2. N. Etemadi, An elementary proof of the strong law of large numbers, Z. Wahrsch. Verw. Gebiete 55 (1981), 119-122.

3. J. Komlós, A generalization of a problem of Steinhaus, Acta Math. Acad. Sci. Hungar. 18 (1967), 217-229.

4. M. Schwartz, Summing subsequences of random variables, Rocky Mountain J. Math. (to appear).

Department of Mathematics, The Ohio State University, Columbus, Ohio 43210 